\title{
Estrategias de estilos de aprendizaje de estudiantes: proceso de validación
}

\author{
Students learning styles strategies: \\ $A$ validation process
}

Dra. Eva Paola Arenas Loera Profesora en la Universidad de Guanajuato (México) (paola.arenas@ugto.mx) (http://orcid.org/0000-0003-4689-687X)

Recibido: 2016-12-07 / Revisado: 2017-04-17 / Aceptado: 2017-04-17 / Publicado: 2017-07-01

\section{Resumen}

A partir de los estilos de aprendizaje se han publicado recomendaciones sobre el uso de ciertas estrategias para los procesos de enseñanza aprendizaje, sin que su funcionamiento haya sido puesto a prueba, en tanto no existe un instrumento que permita su estudio. El objetivo de la investigación fue validar un instrumento de estrategias de aprendizaje basado en los estilos teórico, reflexivo, pragmático y activo, mediante procedimientos de validación de criterio, de constructo, y su corrección psicométrica. La validación por jueces la realizaron investigadores que recomiendan el uso de estrategias de aprendizaje específicas para cada estilo. La validación de constructo se realizó mediante el análisis de los conceptos de estilos de aprendizaje de Alonso, Gallego y Honey (2007), para identificar su congruencia con las estrategias sugeridas. Para identificar el nivel de confiablidad del instrumento, se hizo su aplicación a I 16 estudiantes universitarios. Se obtuvo una confiabilidad general de 0.95 . La confiablidad por factor fue para el estilo activo 0.79 , para el teórico 0.85 , para el reflexivo 0.87 y para el pragmático de 0.88 . Se discute la capacidad de medida para la investigación sobre estrategias asociadas a los estilos de aprendizaje, ya que las recomendaciones deberían basarse en la generación de conocimiento, que favorezca la explicación del funcionamiento de estrategias sobre los procesos de aprendizaje, para tener fundamentación susceptible a verificación.

Descriptores: Estrategias de aprendizaje, instrumento de medida, estilos de aprendizaje, estudiante universitario, proceso de aprendizaje, aplicabilidad de la investigación.

\begin{abstract}
On the basis the dissemination of the use of learning styles, several recommendations have been published on the use of certain strategies, which could be used in teaching-learning process without a way of measuring instrument that allows their study. Thus, the objective of the research was to validate an instrument of learning strategies based on theoretical, reflexive, pragmatic and active styles, through criterion and construct validations, as weel as it's psychometric correction. The criterion validations process was carried out by judges who were researchers that recommended the use of specific learning strategies for each style. The construct validation pro-
\end{abstract}

Forma sugerida de citar: Arenas, E. (2017). Estrategias de estilos de aprendizaje de estudiantes: proceso de validación. Alteridad, 12(2), 224-237. https://doi.org/10.17163/alt.v12n1.2017.08. 
cesss was performed by analyzing the concepts of learning styles by Alonso, Gallego \& Honey (2007) in order to identify their congruence with the suggested strategies. The instrument was answered by 116 college students. The overall reliability obtained was 0.95 . The reliability for the active style was 0.79 , for the theoretical 0.85 , for the reflexive 0.87 and for the pragmatic of 0.88 . The measuring capacity for research on learning styles and strategies, as well as their difussion, are discussed. The results are

\section{Introducción}

La investigación educativa posee características distintivas que le confieren la responsabilidad particular de generar conocimiento y aportar recomendaciones prácticas. Generar conocimiento en el campo educativo, implica reconocer un alto grado de complejidad y diversidad explicativa, susceptible a emplearse pragmáticamente; por eso la información que se publica sobre sus hallazgos debe ser especialmente cuidada. Moreles (2009) identifica tres tipos de publicaciones sobre el campo educativo potencialmente utilizables con fines pragmáticos: documentos estratégicos, ensayos y reportes de investigación.

La utilidad de los reportes de investigación tiene la particularidad de afincarse sobre bases científicas que, como lo expresan Kerlinger y Lee (2008), permiten el desarrollo de conocimiento que además de ser verificable, puede mejorarse en su aplicación práctica. Eso es posible porque sus explicaciones devienen de estructuras teóricas construidas sistemáticamente, se evalúan internamente y son susceptibles a ser probadas. Las pruebas que se hacen son ordenadas, a fin de buscar evidencia empírica mediante relaciones observadas en campo o en laboratorio; son controladas al máximo, con el propósito de distinguirlas a conciencia y sistemáticamente, para descartar explicaciones metafísicas. Gracias al cuidado de este proceso, las explicaciones derivadas poseen la característica particular de autocorregirse. De otra manera, cualquier forma de conocimiento aplicaría indiscriminadamente, su comprensión also discussed on the topic of educational practice, due the recommendations about learning strategies should be based on verificable knowledge and on evidence that explain how are linked the strategies and the learning processes.

Keywords: Learning strategies, measuring instrument, learning styles, university students, learning process, research applicability.

no podría desarrollarse y su autocorrección se dificultaría.

Considerando que la producción científica es evaluada a partir de las publicaciones, que éstas se convalidan mediante su consulta y citación, y que consecuentemente conllevan reconocimiento científico (Cortés, 2007), es necesario cuidar los hallazgos que se publican, pues, en concordancia con Moreles (2009), habrá otros que le encuentren utilidad y los lleve a reproducir y emplear conocimiento o falacias.

El aprendizaje es en concordancia con Cornejo y Redondo (2007), uno de los temas más prolíficamente abordados en publicaciones del campo educativo, ya sea porque permita identificar aspectos optimizadores, o bien para intervenir a manera de orientación y remedio. Respecto a la optimización, por ejemplo, estos estudios contribuyen a comprender el rendimiento académico que. para el caso de la educación superior, representa la posibilidad de potenciar facultades del capital humano.

Relativo a la intervención en procesos de orientación y remedio, el tema se ha centrado en problemas de relevancia socioeducativa, como el rezago, que es un problema persistente porque los alumnos en esta condición suelen ubicarse económicamente entre los menos favorecidos y porque la asignación del servicio educativo, lo brindan generalmente profesores sin la atención requerida (Muñoz, 2009). Otro problema de esta naturaleza es que el mero acceso al sistema educativo, no garantiza que el alumno se mantenga y logre su formación académica (Organización de Estados 
Iberoamericanos, 2010). Entender cuáles y cómo funcionan los factores que, además de incidir en el rezago, mantienen y favorecen la culminación de la formación académica, permitiría contar con explicaciones tanto para optimizar el aprendizaje, como para intervenir en él, mediante procesos de orientación y remedio. En ambos casos se tiene incidencia sobre el rendimiento académico.

La investigación sobre rendimiento académico por medio del aprendizaje, ha permitido el reconocimiento de múltiples variables y distintas dimensiones. Esto es así por la diversidad teórica y áreas de estudio desde las que se aborda (Cornejo y Redondo, 2007), dada la complejidad de la realidad educativa (Albert, 2007).

La presente investigación se enmarca en la tradición que Cornejo y Redondo (2007) categorizan como "estudios de los procesos de enseñanza y aprendizaje escolares basados en las teorías del aprendizaje por "reestructuración" (p. 156). Esta tradición se caracteriza por centrarse en procesos de aprendizaje en el aula, considerados como heterogéneos, ubicados en el constructivismo, en los que no se pretende comparar o jerarquizar a los factores involucrados.

Se desea entender con precisión cómo hace el alumno para aprender considerando procesos derivados de su actividad mental; dicho tópico es tratado en la tradición expuesta, y puede ser entendido a través de ciertas características de los estudiantes. Éstas a su vez, son consideradas por Cerquera (2014), como factores que determinan el rendimiento académico. No se tratarán aquí otros aspectos implicados, porque el interés es encontrar explicaciones sobre cómo las características del alumno, en este caso descritas mediante los estilos de aprendizaje, permiten comprender cómo hace para aprender; a fin de que las recomendaciones prácticas se orienten a que lo haga bajo distintas condiciones, pues en consonancia con Alonso, Gallego y Honey (2007), los estilos de aprendizaje se ubican en enfoques pedagógicos que señalan la necesidad de aprender a aprender, implicando con ello la "efectividad en cualquier situación” (p. 54).
El estudio de los estilos de aprendizaje ha generado diversidad explicativa y por consiguiente pragmática. Su recurrencia ha derivado en la creación de una revista considerada por Soler (2014), como una de las quince publicaciones en español más destacada (también se publica en inglés). Tiene dos eventos internacionales: Congreso Iberoamericano de Estilos de Aprendizaje y Congreso Mundial de Estilos de Aprendizaje (D.J. Gallego, 2015; comunicación personal, 14 de abril de 2015). Asimismo, se ha difundido mediante recursos web 2.0 como: páginas, "blogs, wikis, redes sociales, cursos en línea y foros de discusión" (García, Sánchez, Jiménez y Gutiérrez, 2012, p.68). También son objeto de colaboraciones institucionales en España y México. Esto le da poder de difusión que lo hacen susceptible a utilización práctica o de investigación; tal es el caso de publicaciones que exponen recomendaciones de uso, mediante estrategias de aprendizaje para cada estilo. El problema es que la variedad investigativa desarrollada desde sus orígenes, de acuerdo con Ortiz y Aguilera (2008) dificultan su comprensión y aplicación institucional.

\section{Estilos de aprendizaje}

Ortiz y Aguilera (2008) identificaron dos momentos de desarrollo sobre el tema; el primero mostró que los estilos de aprendizaje se caracterizaban por un componente primordialmente cognoscitivo; el segundo, que fue detonador de las variaciones actuales, se definió por reconocer la existencia de componentes motivacionales que afectan al aprendizaje.

\subsection{Perspectiva de Honey y Mumford}

Honey y Mumford (como se cita en Alonso, Gallego y Honey, 2007) observaron que, ante condiciones de aprendizaje comunes, los alumnos muestran distintas formas de respuesta. Esto llevó a Alonso, et al. (2007) a desarrollar su propuesta subrayando el papel de los alumnos en su aprendizaje, pues son quienes debe dar respuesta 
a esas situaciones, con los recursos de que disponen. Para estos autores, el conocimiento de los estilos de aprendizaje, permite orientar el diseño de la propuesta pedagógica del docente, desde un esquema de aprender a aprender.

Alonso, et al. (2007) definen a los estilos de aprendizaje como "rasgos cognitivos, afectivos y fisiológicos, que sirven como indicadores relativamente estables, de cómo los discentes perciben, interaccionan y responden a sus ambientes de aprendizaje" (2007, p. 48).

Abundando sobre ellos, Gallego y Alonso (2008, como se cita en Arenas, Jiménez y Ávila, 2014), proponen que contienen dos aspectos principales: 1) cognitivo, asociado a la fisiología $y$, por consiguiente, no muestra variaciones a través del tiempo y;2) otro en el que se encuentran las estrategias de aprendizaje, que, al contrario del anterior, es susceptible a variar en función de cómo el individuo las desarrolla, para integrar lo que se va aprendiendo, al estilo predominante. Así, el estilo de aprendizaje es relativamente estable; donde lo "estable" deriva de su base fisiológica y lo "relativo" es producto del aprendizaje y ejercicio de estrategias. Desde este punto de vista, empíricamente se esperaría observar respuestas variables.

En su propuesta también identifican componentes psicológicos o rasgos de los estilos: 1 ) afectivo, que refiere al sentimiento; 2) cognitivo, relativo al conocer y 3) comportamental, que se manifiesta en el hacer. Las funciones de estos componentes son: a) estructurarse con base en el estilo de aprendizaje, b) reflejar la forma en que la persona construye su aprendizaje y c) mostrar mediante estrategias, las respuestas que diseña el individuo, cuando el entorno se lo demande (2008, como se cita en Arenas, et al., 2014).

En el trabajo de Gallego y Alonso (2008, como se cita en Arenas, et al., 2014) no queda clara la distinción entre los aspectos y los componentes psicológicos o rasgos, ni tampoco la relación que tienen entre sí para explicar el funcionamiento de los estilos de aprendizaje, siendo que las estrategias juegan un papel especial, porque su naturaleza relativa al parecer impacta sobre el estilo predominante. Por ejemplo, desde un punto de vista histórico cultural, Ortiz y Aguilera encontraron que la tendencia del estilo solo se mantiene estable, si las condiciones individuales y sociales se mantienen constantes, pero si las condiciones cambian, la persona requerirá plantearse otro esquema a seguir (2010). Estos resultados podrían indicar que las estrategias realizan una especie de "ajuste" en el estilo predominante para enfrentar las situaciones de aprendizaje, mostrando con ello posiblemente que la manifestación de los estilos es tan dinámica, como lo sean las condiciones de aprendizaje. Las estrategias basadas en los estilos son el interés principal de este estudio porque se han desarrollado recomendaciones prácticas, tanto por los autores de esta perspectiva, como de otros investigadores, sin contar con bases que permitan comprender su función.

\subsection{Características de estilos de aprendizaje}

Alonso, Gallego y Honey (2007) afirman que se tienen todos los estilos, pero se tiende más hacia alguno, Sugieren que es ideal el dominio sobre los cuatro, para que se pueda responder a las diferentes situaciones de aprendizaje. Las características generales y específicas para cada estilo según ellos son:

\section{Teórico}

Metódico, lógico, objetivo, crítico y estructurado. Es disciplinado, planificado, sistemático, ordenado, sintético, razonador, pensador, relacionador, perfeccionista, generalizar, buscador de hipótesis, buscador de teorías, de modelos, preguntas, supuestos subyacentes, conceptos, finalidad clara, racionalidad, causas, de sistemas, valores, criterios, inventor de procedimientos, explorador (pp. 73 y 74).

\section{Reflexivo}

Ponderado, concienzudo, receptivo, analítico, exhaustivo, observador, recopilador, pacien- 
te, cuidadoso, detallista, elaborador de argumentos, previsor de alternativas, estudioso del comportamiento, registrador de datos, investigador, asimilador, escritor de informes, lento, distante, prudente, inquisidor, sondeador (pp. 72 y 73).

Pragmático

Experimentador, práctico, directo, eficaz, realista, técnico, útil, rápido, decidido, planificador, positivo, concreto, objetivo, claro, seguro de sí, organizador, actual, solucionador de problemas, aplicador de lo aprendido, planificador de acciones (p.74).

Activo

Animador, improvisador, descubridor, arriesgado, espontáneo, creativo, novedoso, aventurero, renovador, inventor, vital, vividor de la experiencia, generador de ideas, lanzado, protagonista, chocante, innovador, conversador, líder, voluntarioso, divertido, participativo, competitivo, deseoso de aprender, solucionador de problemas, cambiante (p.72).

Aunque las características se encuentran definidas con claridad, se desconoce lo que lleva al estudiante a definir su estrategia. Esto es importante porque su naturaleza relativa, parece sugerir que se supedita a cómo perciben las experiencias de aprendizaje, y probablemente le lleve a ajustar su estilo. Entonces no se sabe si es el dominio de todos los estilos lo que faculta al alumno para atender cualquier situación de aprendizaje o es el manejo que hace sobre sus estrategias. El problema de este desconocimiento, es que se han publicado trabajos con recomendaciones de estrategias para fortalecer todos los estilos, sin contar con evidencia que permita explicar por qué es así.

\section{Estrategias de aprendizaje}

\section{Aprendizaje}

Se comprende aquí el aprendizaje como un proceso constructivo interno, autoestructurante, que depende a su vez del nivel de desarrollo cognitivo; parte de conocimientos previos e implica una reorganización interna, producida cuando el alumno entra en conflicto con lo que ya sabe (Díaz-Barriga y Hernández, 2010).

Estrategias

Por su parte, las estrategias de aprendizaje son un:

Proceso de toma de decisiones, conscientes e intencionales, en los que el alumno elige y recupera, de manera coordinada, los conocimientos conceptuales, procedimentales y actitudinales, necesarios para cumplimentar un determinado objetivo, en función de las condiciones de la situación educativa en que se produce la acción (Juárez, Rodríguez y Luna, 2012, p. 4).

Desde la óptica de la psicología cognitiva, el principal aspecto de la estrategia, es el objetivo, porque permite al aprendiz identificar hacia dónde se dirige el aprendizaje, le lleva a diseñar planes de acción (estrategias) y evaluación, para determinar el grado en que logró su alcance. Los objetivos y la retroalimentación son entonces las principales variables que favorecen el aprendizaje, porque plantean lo que se aprenderá y cómo se hará (uso de estrategias).

Las funciones de las estrategias son: facilitación cognitiva del aprendizaje, adquisición del conocimiento en general y promoción de logro de aprendizaje significativo. Los supuestos también consideran que cada persona es única, por lo que sus formas de aprender son diferentes (Lago, Colvin, y Cacheiro, 2008). En este sentido se sugiere que cada persona puede emplear estrategias de manera particular, sin tener claro en función de qué sucede esto.

\section{Estilos y estrategias de aprendizaje}

Alonso, Gallego y Honey (2007) consideran que los estilos dan cuenta de características que los alumnos usan para responder a situaciones de aprendizaje, afirmando que es deseable contar con altos niveles en todos para responder a distintas condiciones de aprendizaje. En su libro 
Estilos de Aprendizaje incluso dedican espacios para describir formas de cómo mejorar cada estilo y recomendar pautas de acción tutorial, pero nuevamente no se tienen datos sobre cómo funciona o cómo se explica. Esto impulsó publicaciones con recomendaciones prácticas en este tenor, aún sin tener claro si son los estilos per se los que definen la forma de responder al aprendizaje, o alguna clase de ajuste entre sus componentes; por ejemplo, en función de cómo operan las estrategias. La consecuencia de ello es que no se logra demostrar el impacto de su implementación (Aragón y Jiménez, 2009), incluso para los usuarios de los estilos pueden no ser relevantes (Castro y Guzmán, 2005).

Ejemplos de esta productividad son las publicaciones que muestran propuestas de estrategias de aprendizaje derivadas de la identificación de estilos del alumnado. Reinicke, Chiang, Montecinos, Del Solar, Madrid y Acevedo (2008) lo hicieron para estudiantes de Ciencias Biológicas; Ortega (2008) de Odontología; Medina y Medina (2012) y Giménez-Bertomeu, de Alfonseti Hartmann, Lillo, Lorenzo, Mira-Perceval, Rico y Asensi (2008) para Trabajo Social; Jiménez (2004) realizó un estudio intercultural con estudiantes extranjeros. Estos autores recomiendan integrar los estilos de aprendizaje en procesos educativos, pero no muestran los resultados de su implementación.

En este ánimo de buscar mecanismos para el logro de los aprendizajes, en un estudio anterior a este, se hizo un perfil de casos de estilos de aprendizaje y uso de estrategias en alumnos de genómica alimentaria e innovación educativa (Arenas, Reyes y Ávila, 2014). Aunque cualitativamente se encontró que su comportamiento para estrategias de activación, procesamiento de información, metacognición, organización y aplicación, fue acorde con las características propias de su estilo, el aprendizaje logrado respecto al material propuesto fue pobre. Reconocieron la importancia de las estrategias en su aprendizaje, pero no las utilizaron. En este estudio particular no se logra comprobar el impacto de los estilos, sobre el aprendizaje. Si se piensa que las estrategias tienen un papel esencial en la manifestación de los estilos y que estos estudiantes no las emplearon, posiblemente ahí se encuentre parte de la explicación, pero no se puede verificar en tanto que no se teorizado o encontrado evidencia empírica, al respecto.

A pesar de ello algunos autores desarrollaron estrategias concretas sin obtener resultados concluyentes que los lleva a insistir en recomendar su empleo como parte de las estrategias de enseñanza (Del Valle de Moya, Hernández, Hernández y Cózar, 2009; Farfán, Gallardo, Terán y Alonso, 2010); otros aseveran que su uso favorece la calidad educativa (Ventura, 2011); se han expuesto estrategias asociadas a estilos identificados (Cózar, Bravo y Fernández, 2012); se afirma que promueven el aprendizaje y que su identificación permite ubicar grupos de riesgo de alumnos de bajo rendimiento, así como nivel de accesibilidad del contenido de las asignaturas (Del Valle de Moya, et al., 2009); pero ninguno de estos trabajos comprueba, y por consiguiente no explica la incidencia de los estilos sobre el aprendizaje.

El interés por los estilos de aprendizaje ha favorecido la producción de propuestas de estrategias asociadas a ellos, por buscar mejoras en la práctica educativa; pero lo prolífica que pueda ser la productividad sobre un campo o tema, no da cuenta de su capacidad explicativa. Aunque se tienen claramente caracterizados en este caso por Alonso, et al. (2007), su conceptualización es diversa, sus componentes confusos y no explica qué es lo que define las respuestas ante las situaciones de aprendizaje.

Para conocer si las estrategias difundidas pueden ser fundamentadas sobre la base de los estilos de aprendizaje, se propuso desarrollar un instrumento que permitiera estudiarlas. Se esperaba que este instrumento permitiera precisar la posibilidad de identificar estrategias de aprendizaje asociadas a cada estilo. También se pretende que el instrumento permita explorar el funcionamiento de estas estrategias, para saber si 
es posible sentar bases que fundamenten propuestas sobre su uso.

$\mathrm{Al}$ hacer recomendaciones prácticas sobre estrategias susceptibles a ser examinadas de forma sistemática y puestas a prueba, se da pauta a la replicabilidad y se favorece la autocorrección; promoviendo así el desarrollo de conocimiento sobre estrategias de estilos de aprendizaje, mediante la acción investigativa, para que su utilización práctica ofrezca bases explicativas. De esta manera el objetivo de la investigación fue validar un instrumento de estrategias de aprendizaje basadas en los estilos teórico, reflexivo, pragmático y activo.

\section{Método}

Inicialmente, a partir de los trabajos de Farfán, et al. (2010), Del Valle de Moya, et al. (2009) y Cózar, et al. (2012) se pudieron observar estrategias propuestas para fortalecer los estilos de aprendizaje. Las primeras se dirigían a la estimulación del desarrollo de estilos bajos; las segundas y terceras eran tareas personalizadas para cada estilo. Con estas estrategias se diseñaron 90 reactivos que se utilizaron para la primera aplicación.

Posteriormente, conforme se avanzó en la validez de constructo, se siguieron las características y formas de aprender generadas por Alonso, et al. (2007), de manera que para la segunda aplicación, quedaron 91 reactivos.

\section{Procedimiento}

\section{Fase 1. Validez de criterio}

Se invitó, vía correo electrónico, a ocho de los autores de las estrategias y estilos de aprendizaje como jueces. Siete aceptaron participar en esta fase del proceso. Se les facilitó un formato de revisión, un instructivo y el instrumento. Su tarea consistió en comparar cada reactivo con los aspectos y características de cada estilo. Para comparar debieron considerar: la identificación de la congruencia de cada reactivo con cada estilo, el acuerdo o desacuerdo en que el reactivo sirvie- ra para evaluar estrategias, la determinación de si cada reactivo en su formulación expresaba de forma adecuada la una estrategia de aprendizaje fundamentada en su estilo; si el contenido de cada reactivo tenía coherencia con los principios de los estilos de aprendizaje, su apreciación sobre a qué estilo de aprendizaje correspondía cada reactivo, la verificación de si la redacción del reactivo era comprensible, la opinión final para establecer si el reactivo se conservaba, eliminaba o modificaba. Los académicos revisaron cada uno de los reactivos, respondiendo a cada tarea planteada.

Fase 2. Validez de constructo

Para esta fase resultó esencial la participación de un octavo juez, que sugirió que el instrumento se contrastara con la publicación sobre los estilos de aprendizaje de Alonso, et al. (2007) en la cual tiene coautoría. A partir de esta sugerencia, dos académicos que colaboran desde diferentes ciudades y de manera conjunta, para desarrollar propuestas psicopedagógicas en sus respectivas facultades, y quienes tienen un interés compartido en los estilos de aprendizaje (uno perteneciente a un programa de psicología y otro a uno de psicología y otro de arquitectura), contrastaron los reactivos, haciendo el análisis de contenido de la propuesta de estilos de aprendizaje. Con esa base se seleccionaron y agruparon a doble ciego los reactivos que concordaban con los aspectos y características de cada estilo de aprendizaje, así como con el apartado que aborda el tema de formas para mejorar cada estilo. Tras la comparación de las observaciones obtenidas, se discutió y se acordó la clasificación final de los reactivos, ajustándose también su redacción para que se caracterizaran como estrategias, esto es, incluyendo el objetivo a seguir y la acción que correspondería para alcanzarlo, con el propósito distinguirlas de los estilos de aprendizaje.

Con el instrumento reconocido en su estructura conceptual fue aplicado a una muestra no probabilística por conveniencia de 116 universitarios voluntarios, de los cuales 59\% fueron mujeres y $41 \%$ hombres con edad promedio de 21 años $(\mathrm{DE}=4.5)$. La mayoría $(\mathrm{n}=90)$ fue alum- 
nos de los programas de psicología y el resto de arquitectura $(n=26)$ a los que pertenecían los académicos que hicieron la revisión final. Este suministro permitió la corrección psicométrica del instrumento. Aunque no se buscaba un estudio comparativo entre licenciaturas, las carreras de psicología y arquitectura podrían ser equivalentes a las carreras experimentales y ciencias de la salud, así como humanidades clasificadas por Alonso (1992, como se cita en Alonso, et al., 2007) para interpretar sus propios datos.

El instrumento volvió a aplicarse a una muestra de 263 estudiantes de psicología, con alrededor de 21 años $(\mathrm{DE}=3.1)$ de los cuales la mayoría eran mujeres (71\%), siendo el $29 \%$, hombres.

\section{Resultados}

\section{Validez de criterio}

En la validez de criterio en general las observaciones se hicieron sobre la redacción de las instrucciones y algunos reactivos. Solo una de las investigadoras señaló que era preciso revisar el libro de Alonso, et al. (2007). Ninguno sugirió cambios que modificaran en forma importante el instrumento. Como producto de las concordancias se eliminaron tres reactivos de la escala (41, 48,71 ), quedando así 87 reactivos (ver Tabla 1).

Tabla 1. Agrupación de reactivos según estrategias

\begin{tabular}{|c|c|}
\hline Estrategias para cada estilo & Reactivos \\
\hline Pragmáticas & $\begin{array}{l}1,4,13,17,18,19,22,24,27,37,45,49,57,69,72,82,84,85,86 \text {, } \\
88,89\end{array}$ \\
\hline Activas & $7,8,10,15,16,21,23,42,51,52,55,58,59,60,79,81,87,90$ \\
\hline Teóricas & $\begin{array}{l}2,3,12,14,26,30,31,35,39,43,54,61,62,64,66,70,73,74,76 \text {, } \\
77,78,80,83\end{array}$ \\
\hline Reflexivas & $\begin{array}{l}5,6,9,11,20,25,28,29,32,33,34,36,38,40,44,46,47,50,53 \\
56,63,65,67,68,75\end{array}$ \\
\hline
\end{tabular}

Validez de constructo

Respecto a la validez de constructo se encontraron concordancias entre las estrategias de aprendizaje exploradas por el instrumento, con las características de los estilos de aprendizaje y formas para aprender de Alonso, et al. (2007). Esto permitió que los reactivos eliminados se reintegraran, notándose que uno de estos reactivos indagaba dos aspectos al mismo tiempo, por lo que se decidió dividirlo en dos. De esta manera quedaron 91 reactivos en total. A continuación se presenta el comparativo para cada estilo.

Las características de alumnos con estilo pragmático son: organizador, planificador de acti- vidades, aplicador de lo aprendido, solucionador de problemas, concreto, actual, práctico, técnico, experimentador, directo, eficaz, claro, realista, útil, decidido, positivo. Sus formas de aprender son: estar expuesto ante modelo que pueda emular, tener posibilidad inmediata de aplicar lo aprendido, elaborar planes de acción con resultado evidente, dar indicaciones sugerir atajos, experimentar o practicar con asesorías de expertos, ver nexo entre tema y problema u oportunidad para aplicarlo, ver demostraciones con expertos o reconocidos, ver ejemplos o anécdotas, concentrarse en aspectos prácticos, comprobar que la actividad tiene validez inmediata, experimentar simulaciones, comprobar que expertos dominan 
el tema y saben hacer las cosas. En la Tabla 2 se pueden observar las características generales de las estrategias asociadas a este estilo.

Tabla 2. Comparación entre estilo pragmático con las estrategias de aprendizaje

\begin{tabular}{|c|c|c|}
\hline Dimensión & \multicolumn{1}{c|}{$\begin{array}{c}\text { Características generales } \\
\text { Estrategias de Aprendizaje }\end{array}$} \\
\hline Pragmática & $\begin{array}{l}\text { Plantear objetivos sobre la identificación de situaciones reales para definir } \\
\text { los pasos que permitan su atención }\end{array}$ \\
\hline $\begin{array}{l}\text { Encontrar la utilidad práctica de lo que revisa en clase } \\
\text { - }\end{array}$ & $\begin{array}{l}\text { Actualizar lo que sabe y cómo se hacen las cosas con expertos } \\
\text { su utilidad } \\
\text { Dirigir a otros para desarrollar tareas }\end{array}$ \\
\hline
\end{tabular}

Alumnos con estilo activo son: novedoso, participativo, lanzado, protagonista, conversador, divertido, líder, innovador, creativo, novedoso, inventor, deseoso de aprender, solucionador de problemas, vividor de la experiencia, vital, generador de ideas, competitivo, voluntarioso, chocante, aventurero, renovador, espontáneo. Sus formas de aprender contemplan: hacer cosas nuevas, generar nuevas ideas, resolver problemas, vivir situaciones de interés o crisis, hacer presentaciones, intervenir activamente, arriesgarse, resolver como parte de equipos, resolver algo que no sabía o no podía, encontrar dificultades, tener con quien dialogar, no quedarse sentado por mucho tiempo, intentar algo diferente. Las características de las estrategias para este estilo se observan en la Tabla 3.

Tabla 3. Comparación entre estilo activo con las estrategias de aprendizaje

\begin{tabular}{|c|c|c|}
\hline Dimensión & \multicolumn{1}{c|}{$\begin{array}{c}\text { Características generales } \\
\text { Estrategias de Aprendizaje }\end{array}$} \\
\hline Activa & $\begin{array}{l}\text { Utilizar diversas y dinámicas formas de aprender para mantener su parti- } \\
\text { cipación } \\
\text { Participar activamente en distintas iniciativas que involucren la movilización } \\
\text { de otros y su protagonismo }\end{array}$ \\
\hline $\begin{array}{l}\text { Utilizar habilidades sociales para conseguir lo que se propone } \\
\text { Simplificar formas de hacer cosas } \\
\text { Seguir los propios esquemas sin intervención de otros }\end{array}$ \\
\hline
\end{tabular}

Se describe al alumno con estilo teórico con las siguientes características: crítico, relacionador, sintético, estructurado, ordenado, disci- plinado, planificado, sistemático, perfeccionista, metódico, organizado. Sus formas de aprender consideran: sentirse en situaciones estructuradas 
con finalidad clara, inscribir la información en sistemas modelos y teorías, analizar la situación completa, encontrar ideas complejas capaces de interesarle. Las características de las estrategias asociadas a este estilo se ilustran en la Tabla 4.

Tabla 4. Comparación entre estilo teórico con las estrategias de aprendizaje

\begin{tabular}{|c|ll|}
\hline Dimensión & \multicolumn{1}{c|}{$\begin{array}{c}\text { Características generales } \\
\text { Estrategias de Aprendizaje }\end{array}$} \\
\hline Teóricas & - $\begin{array}{l}\text { Contar con representaciones de información organizada de manera clara y sintética } \\
\text { - Tener información disponible con apoyo de distintos mecanismos }\end{array}$ \\
& $\begin{array}{l}\text { Relacionar información formando esquemas de explicación panorámicos } \\
\text { - Tener elementos para conservar una visión crítica sobre las cosas } \\
\text { que ésta se almacenan }\end{array}$ \\
\hline
\end{tabular}

Los alumnos con estilo reflexivo se caracterizan por ser: analítico, observador, recopilador, registrador de datos, exhaustivo, escritor de informes, elaborador de argumentos, previsor de alternativas, sondeador, concienzudo, cuidadoso, detallista, lento, asimilador, receptivo, investigador, prudente. Sus formas de aprender son: reflexionar sobre actividades Intercambiar opi- niones, trabajar sin presión de tiempo, investigar detenidamente, reunir información, pensar antes de actuar, asimilar antes de comentar, hacer análisis detallados realizar informes cuidadosamente ponderados. En comparación, las características de las estrategias reflexivas se muestran en la Tabla 5.

Tabla 5. Comparación entre estilo reflexivo con las estrategias de aprendizaje

\begin{tabular}{|c|c|c|}
\hline Dimensión & \multicolumn{1}{c|}{$\begin{array}{c}\text { Características generales } \\
\text { Estrategias de Aprendizaje }\end{array}$} \\
\hline Reflexiva & $\begin{array}{l}\text { Generar ideas propias tras el análisis exhaustivo de sus pensamientos en contraste } \\
\text { con los de otros. } \\
\text { Contar con todos los recursos disponibles antes de avanzar con seguridad } \\
\text { Argumentar con base en aspectos derivados de distintos ángulos y de las propias } \\
\text { apreciaciones, para explicar las cosas } \\
\text { Difundir el conocimiento adquirido tras una laboriosa y extensa investigación sobre } \\
\text { temas novedosos }\end{array}$ \\
\hline
\end{tabular}

Las Tablas 2 a 5 contienen una síntesis de las características del conjunto de estrategias para cada estilo de aprendizaje contenidas en el instrumento. Su concordancia se identificó analizando el contenido de los reactivos en contraste con las características de los estilos de aprendizaje y formas de aprender de Alonso, et al. (2007).

Análisis de confiabilidad

En un primer momento se realizó el análisis de fiablilidad de los reactivos mediante el 
coeficiente alpha de Cronbach. El Alpha total, de los 87 reactivos fue de 0.95 , mostrando con ello consistencia interna. Posteriormente se realizó el mismo análisis para cada conjunto de estrategias. Los resultados de confiabilidad y ejemplos de algunos reactivos se pueden ver en la Tabla 6 .

Tabla 6. Análisis de confiabilidad por indicador ejemplificado con algunos reactivos

\begin{tabular}{|c|c|c|c|}
\hline $\begin{array}{l}\text { Estrategias para } \\
\text { cada estilo }\end{array}$ & a & $\begin{array}{l}\text { Cantidad de } \\
\text { Reactivos }\end{array}$ & Reactivos \\
\hline Pragmáticas & 0.88 & 21 & $\begin{array}{l}\text { - Identifico actividades donde se puede poner en práctica lo que } \\
\text { aprendo en clases } \\
\text { - Identifico problemas socialmente relevantes relacionados con los } \\
\text { temas de clase } \\
\text { - Escucho, por mi cuenta, conferencias con profesionales de la ca- } \\
\text { rrera de mi interés }\end{array}$ \\
\hline Activas & 0.79 & 18 & $\begin{array}{l}\text { - Elaboro frases y/o acrósticos para estudiar conceptos que se me } \\
\text { dificultan } \\
\text { - } \quad \text { Participo en clase con mis propias ideas aún sin la petición del } \\
\text { profesor } \\
\text { - Busco información en internet más que en la biblioteca }\end{array}$ \\
\hline Teóricas & 0.85 & 23 & $\begin{array}{l}\text { - } \quad \text { Elaboro mapas conceptuales como una actividad de estudio } \\
\text { - } \quad \text { Cuido la estructura, orden y limpieza en los documentos que elabo- } \\
\text { ro para la escuela } \\
\text { - Me esmero en obtener buenas calificaciones parciales para que no } \\
\text { se cargue el trabajo al final de los cursos }\end{array}$ \\
\hline Reflexivas & 0.87 & 25 & $\begin{array}{l}\text { - Incluyo mis propias reflexiones y conclusiones cuando realizo tra- } \\
\text { bajos escolares } \\
\text { - } \quad \text { Planteo mis dudas en clase al profesor } \\
\text { - } \quad \text { Tomo apuntes personales de las lecturas que realizo }\end{array}$ \\
\hline
\end{tabular}

Tras la segunda aplicación se obtuvo una confiabilidad total de 0.94 para los 91 reactivos resultando que la consistencia interna para cada conjunto quedó para las estrategias de estilo pragmático de 0.86 ; para las activas, 0.79 ; para las teóricas, 0.86 y para las reflexivas, 0.83 .

\section{Discusión y conclusiones}

En general el instrumento tiene capacitad de utilización. La validez de criterio facilitó precisar instrucciones y redactar los ítems con legibilidad para ser comprendidos como estrategias y no como actividades indicadas por los profesores, en tanto que cada reactivo reflejaba con la mayor claridad posible que contenía un objetivo y una acción encaminada a conseguirlo. El instrumento mostró estabilidad tras su verificación en la segunda aplicación.

Las estrategias recomendadas en las publicaciones de Farfán, et al. (2010), Del Valle de Moya, et al. (2009) y Cózar, et al. (2012), se inspiraron en su momento en las propuestas de Alonso, et al. (2007), pero no se constató si tales estrategias podrían fundamentarse sobre la base de los estilos de aprendizaje. Por esta razón en 
el presente trabajo se esperaba saber si con un instrumento era propicio estudiar estrategias asociadas a cada estilo, para poder fundamentarlas y explicarlas a partir de ellos. Los resultados mostraron que el instrumento identifica estrategias asociadas a los estilos, por lo que sí es posible tener estrategias de aprendizaje que puedan explicarse a través de ellos.

Esto permite que el alumno pueda reconocer sus propias estrategias, las pueda complementar con otras que correspondan a su estilo y también le permita identificar otras estrategias para fortalecer estilos en los que se encuentre bajo. Por ahora el estudio se limitó a comprobar la correspondencia entre recomendaciones prácticas inspiradas en la propuesta de los estilos de aprendizaje; se logró la comprobación tras su aplicación en alumnos de dos programas de licenciatura y se constataron los resultados en una segunda aplicación. Se considera que el instrumento es potencialmente utilizable porque siendo los participantes de distintas ciudades, su consistencia se mantuvo estable.

Se observó que las estrategias publicadas dejaron fuera algunas características específicas y formas de aprender de los cuatro estilos de aprendizaje de Alonso (et al). Eso demuestra el punto que se trata aquí acerca del cuidado que se necesita tener cuando se difunden recomendaciones prácticas.

En general las características y formas de aprender de Alonso, et al. (2007) son consistentes con los reactivos del instrumento y entre sí, lo cual se observó con la confiablidad total y por indicador en las dos aplicaciones. El proceso de validez de criterio y constructo se desarrolló con sistematicidad, control y verificación, en tanto que se siguieron pasos claros para cada parte. El trabajo fue seguido y verificado por diez académicos y probado con dos aplicaciones. Se favoreció el avance del conocimiento sobre estrategias asociadas a estilos de aprendizaje recomendadas en publicaciones porque se cuenta con evidencia que muestra concordancia teórico-práctica a través de la revisión de insumos en esas dos dimensiones, posibilitando así la autocorrección (Kerlinger y Lee, 2008).

El contacto directo con los autores de las propuestas reconoce plenamente su trabajo, y su esfuerzo brinda la posibilidad del avance del conocimiento. Este es parte de reconocimiento y convalidación a la que Moreles (2009) aludía; su accesibilidad y disponibilidad para realizar el trabajo hizo posible autocorregir la laguna faltante, ya que de acuerdo con Kerlinger y Lee (2008), al buscar un sistemático, concienzudo y ordenado manejo de la información en un campo, se está en condiciones de obtener explicaciones que brinden fundamentación en la acción investigativa para el avance del conocimiento y su recomendación práctica.

\section{Referencias bibliográficas}

Albert, J. (2007). La investigación educativa. Claves teóricas. Madrid: McGraw-Hill.

Alonso, C., Gallego, D., \& Honey, P. (2007). Los estilos de aprendizaje. Bilbao: Mensajero.

Aragón, M., \& Jiménez, Y. (2009). Diagnóstico de los estilos de aprendizaje en los estudiantes: Estrategia docente para elevar la calidad educativa. CPU-e, Revista de Investigación Educativa, 9, julio-diciembre. Recuperado de https://goo.gl/Pw2mxz

Arenas, E., Jiménez E., \& Ávila, J. (2014). Estilos de Aprendizaje en alumnos de Innovación Educativa. Revista Congreso Universidad, 3(2). Recuperado de https://goo.gl/aVEL57

Arenas, E., Reyes, M., \& Ávila, J. (2014). Estilos y estrategias de aprendizaje en estudiantes universitarios. Trabajo presentado en el VIII Coloquio de Humanidades. Diálogos sobre Cultura, Arte y Sociedad. Facultad de Filosofía y Letras de la Universidad Autónoma de Nuevo León. Recuperado de https://goo.gl/3ymbCQ

Castro, S., \& Guzmán, B. (2005). Los estilos de aprendizaje en la enseñanza y el aprendizaje: una propuesta para su implementación. Revista de Investigación, 58, 83-102. Recuperado de https://goo.gl/4aS5oy

Cerquera, O. (2014). Estado del arte del rendimiento académico en la educación media. Rhec. 
17(17), 197-220. Recuperado de https://goo. $\mathrm{gl} / 5 \mathrm{~g} 81 \mathrm{jZ}$

Cornejo, R., \& Redondo, J. (2007). Variables y factores asociados al aprendizaje escolar. Una discusión desde la investigación actual. Estudios Pedagógicos, 33(2), 155-175. Recuperado de https://goo.gl/Uxng1c

Cortes, D. (2007). Medir la producción científica de los investigadores universitarios: la bibliometría y sus límites. Revista de Educación Superior, 2(142), 43-65. Recuperado de https://goo.gl/B3uNAH

Cozar, R., Bravo, R., \& Fernández, A. (2012). Estrategias educativas personalizadas para cada estilo de aprendizaje. Trabajo presentado en el V Congreso Mundial de Estilos de Aprendizaje. Santander. Recuperado de https://goo.gl/zWiF5Q

Díaz Barriga, F., \& Hernández, G. (2010). Estrategias docentes para un aprendizaje significativo. México: McGraw Hill.

García, J., Sánchez, C., Jiménez, M., \& Gutiérrez, M. (2012). Estilos de Aprendizaje y Estrategias de Aprendizaje: un estudio en discentes de postgrado. Revista Estilos de Aprendizaje, 10(10), 1-17. Recuperado de https://goo.gl/6dfjpQ

Giménez-Bertomeu, V., \& Alfonseti de Hartmann, N., Lillo, A., Lorenzo, J. Mira-Perceval, M., Rico, J. y Asensi, M. (2008). Estilos de aprendizaje y método de caso en Trabajo Social. Revista Estilos de Aprendizaje, 2(2), 1-23. Recuperado de https://goo.gl/QXDLVU

Farfán, S., Gallardo, R., Terán, J., \& Alondo, C. (2010). Aplicación de los estilos de aprendizaje para la determinación de grupos de riesgo en la carrera de Informática de la UMSA. Revista de Estilos de Aprendizaje, 6(6), 1-17. Recuperado de https://goo.gl/VQCDe9

Jiménez, H. (2004). El impacto de las diferencias de los estilos de aprendizaje interculturales en la enseñanza a los alumnos extranjeros de intercambio del tec. Trabajo presentado en la Reunión de Intercambio de Experiencias en Estudios de Educación en el Tecnológico de Monterrey. Recuperado de https://goo.gl/dDtWBN
Juárez, C., Rodríguez, G., \& Luna, E. (2012). El cuestionario de estilos de aprendizaje CHAEA y la escala de estrategias de aprendizaje ACRA como herramienta potencial para la tutoría académica. Revista Estilos de Aprendizaje, 10(10), 1-31. Recuperado de https://goo.gl/bfgD15

Kerlinger, F., \& Lee, H. (2008). Investigación del comportamiento. Métodos de Investigación en ciencias sociales. México: McGraw-Hill.

Lago, B., Colvin, L., \& Cacheiro, M. (2008). Estilos de aprendizaje y actividades polifásicas: Modelo EAAP. Revista Estilos de aprendizaje, 2(2), 1-24. Recuperado de https://goo.gl/NsB9Yj

Medina, M., \& Medina, E. (2012). Evaluación Estilos de Aprendizaje en Trabajo Social. Trabajo presentado en el V Congreso Mundial de Estilos de Aprendizaje. Santander. Recuperado de https://goo.gl/t4xaSD

Moreles, J. (2009). Uso de la Investigación Social y Educativa. Recomendaciones para la agenda de investigación. Revista Perfiles Educativos, 21(124), 93-106. Recuperado de https://goo.gl/AUJJhR

Muñoz, C. (2009). Construcción del Conocimiento sobre la Etiología del Rezago Educativo y sus Implicaciones para la Orientación de las Políticas Públicas: la Experiencia de México. Revista Iberoamericana sobre calidad y Cambio en Educación, 7(4), 10-27. Recuperado de https://goo.gl/Mniy9n

OEI (2010). Metas Educativas 2021. La Educación que queremos por la generación de los bicenterarios. Recuperado de https://goo.gl/LxQ3yZ

Ortega, L.C. (2008). Estilos de aprendizaje en los estudiantes de odontología de la UACJ. (Tesis de Maestría). Universidad de Ciencias Aplicadas. Colombia. Recuperado de https://goo.gl/xKbNWV

Ortiz, E., \& Aguilera, E. (2008). La caracterización de perfiles de estilos de aprendizaje sus implicaciones didácticas en la educación superior. Revista Pedagogía Universitaria, 8(5). Recuperado de https://goo.gl/g8Hza3

Ortiz, E., \& Aguilera, E. (2010). El estudio de perfiles de los estilos de aprendizaje en estudiantes Universitarios, desde la concepción histórico-cultural de L.S. Vigotski. Revista Pedagogía Universitaria, 15(3). Recuperado de https://goo.gl/Tdzepf 
Reinicke, K., Chiang, M. Montecinos, H., Solar del, M., Madrid, V., \& Acevedo, C. (2008). Estilos de aprendizaje de alumnos que cursan asignaturas de Ciencias Biológicas en la Universidad de Concepción. Revista Estilos de Aprendizaje, 2(2), 170-181. Recuperado de https://goo.gl/4ojpC8

Soler, M. (2014). El constructo Enfoques de Aprendizaje: un análisis bibliométrico de las publicaciones en español en los últimos 20 años. Revista Colombiana de Educación, 66, 127-148, enero-junio. Recuperado de https://goo.gl/jy73J4
Valle del Moya, M., Hernández J. Hernández J., \& Cozar, R. (2009). Un estilo de aprendizaje, una actividad. Diseño de un plan de trabajo para cada estilo. Revista Estilos de Aprendizaje, 4(4), 1-16. Recuperado de https://goo.gl/ZuzbXY

Ventura, A. (2011). Estilos de aprendizaje y prácticas de enseñanza en la universidad. Un binomio que sustentan la calidad educativa. Perfiles Educativos, 33(núm-esp), 142-154. Recuperado de https://goo.gl/qWjDMi 